\title{
Core-Shell Engineering to Enhance the Spectral Stability of Heterogeneous Luminescent Nanofluids
}

\author{
Lucía Labrador-Páez, Marco Pedroni, Krisjanis Smits, Adolfo Speghini, \\ Francisco Jaque, José García-Solé, Daniel Jaque, * and Patricia Haro-González
}

The tendency to the miniaturization of devices and the peculiar properties of the nanoparticles have raised the interest of the scientific community in nanoscience. In particular, those systems consisting of nanoparticles dis-persed in fluids, known as nanofluids, have made it possible to overcome many technological and scientific challenges, as they show extraordinary properties. In this work, the loss of the spectral stability in heterogeneous luminescent nanofluids is studied revealing the critical role played by the exchange of ions between different nanoparticles. Such ion exchange is favored by changes in the molecular properties of the solvent, making heterogeneous luminescent nanofluids highly unstable against temperature changes. This work demonstrates how both temporal and thermal stabilities of heterogeneous luminescent nanofluids can be substantially improved by core-shell engineering This simultaneously avoids the leakage of lumines-cent ions and the effects of the solvent molecular changes.

\section{Introduction}

Nanofluids (NFs, colloidal dispersions of nanoparticles in fluids) are attracting the attention of the scientific community due to their versatility and unique properties. ${ }^{[1]}$ In fact, NFs have already allowed, for instance, to increase the thermal conductivity of fluids, ${ }^{[2]}$ in vivo fluorescent imaging, ${ }^{[3]}$ and to study the fundamentals of the Brownian motion of nanoparti-cles. ${ }^{[4]}$ In particular, those nanofluids consisting of two or more types of nanoparticles with different properties dispersed in fluids, known as heterogeneous nanofluids, are nowadays of great interest due to their outstanding multifunctionality. For instance, heterogeneous nanofluids consisting in nanoheaters and nanothermometers are being used in controlled hyperthermia treatments, ${ }^{[5]}$ a heterogeneous nanofluid composed of gold nanorods of diverse aspect ratios allows multiplexed plasmonicbiosensing, ${ }^{[6]}$ or a heterogeneous nanofluid with donor and acceptor nanoparticles enables to apply Förster resonant energy transfer to diagnosis. ${ }^{[7]}$ Additionally, het-erogeneous nanofluids have allowed to study new physical phenomena of energy transfer between nanoparticles. ${ }^{[8]}$

However, recent studies demonstrated how the spectral properties of heteroge-neous luminescent nanofluids (HLNFs) can be undesirably affected by disrupting processes originating the spectral stability loss such as ion exchange between the diverse luminescent nanoparticles (LNPs). ${ }^{[9]}$ Ion exchange (i.e., transfer

of ions between two different chemical entities) has been traditionally treated as a positive effect. For instance, it has been used not only as a synthesis route for engineering in nanoscience, ${ }^{[10]}$ but it has also been employed as biosensing strategy. ${ }^{[11]}$ Nevertheless, ion exchange between the LNPs in the HLNFs is disadvantageous as it could imply the loss of the multifunctionality in HLNFs, i.e., ion exchange could modify the LNPs constituting the HLNF, resulting in the loss of its heterogeneity. Therefore, a strategy to prevent the destabili-zation of the HLNFs due to interparticle ion exchange (IPIE) should be identified. As evidenced by previous works, IPIE is a surface effect, i.e., only the outer ions may be exchanged among the LNPs. ${ }^{[10]}$ Consequently, the effect of ion exchange 
on the emission of the LNPs would be more easily noticeable for smaller LNPs, as the surface/volume ratio increases. As it is a surface effect, a surface passivation by means of a nonluminescent shell would prevent the IPIE in the HLNF and thus the loss of its heterogeneity. Core-shell engineering has been widely employed so far to overcome diverse challenges in nanoscience. ${ }^{[8 \mathrm{a}, 12]}$ For instance, LNPs used for photothermal biological treatments can be self-monitored by the addition of a temperature-sensitive shell ${ }^{[12 b]}$ and drastic improvements of luminescence efficiency have also been achieved by avoiding undesirable quenching processes. ${ }^{[12 \mathrm{~d}]}$

The evolution of the IPIE process in HLNFs is strongly determined by the interaction between the fluid and the LNPs. In particular, in water-based nanofluids, its molecular behavior at around $45{ }^{\circ} \mathrm{C}$ has been proved to affect drastically the rate at which IPIE is taking place. ${ }^{[9]}$ Below that temperature, water molecules are able to interact between them to form sort of cages around the LNPs that isolate them and make the IPIE process less effective. One the other hand, at higher tempera-tures the molecular state of water favors IPIE so that the lumi-nescent properties of the HLNFs become strongly unstable against temperature changes. The use of core-shell engineering to prevent the IPIE in HLNFs and, in particular, to avoid time and temperature instabilities, has not yet been explored.

In this work, a core-shell structure is used as surface passivating agent to prevent the IPIE and so to keep the temporal and thermal spectral stability of HLNFs. This strategy is proved using $\mathrm{SrF}_{2}: \mathrm{Nd}$ and $\mathrm{SrF}_{2}: \mathrm{Yb}$ LNPs simultaneously dispersed in water or $\mathrm{D}_{2} \mathrm{O}$ to form HLNFs. The IPIE taking place in these HLNFs is monitored by means of the energy transfer from $\mathrm{Nd}^{3+}$ (donors) to $\mathrm{Yb}^{3+}$ (acceptors) ions. The effect of the interac-tion between the solvent molecules on the IPIE process and its dependence on temperature are also analyzed in this work.

\section{Results and Discussion}

The studied HLNF consists of $\mathrm{SrF}_{2}: \mathrm{Nd}$ (diameter of $12.4 \mathrm{~nm}$ ) and $\mathrm{SrF}_{2}: \mathrm{Yb}(8.9 \mathrm{~nm}) \mathrm{LNPs}$ dispersed in water (see Figure 1). The lack of spectral stability of this HLNF is pre-vented by adding a nonluminescent shell made of $\mathrm{SrF}_{2}$ : $\mathrm{Y}$ to the surface of the LNPs (LNPs@Y), resulting in a core-shell HLNF (HLNF@Y). The HLNF@Y consists of $\mathrm{SrF}_{2}: \mathrm{Nd} @ \mathrm{SrF}_{2}: \mathrm{Y} \quad\left(\begin{array}{ll}20 & \mathrm{~nm}\end{array}\right)$ and $\mathrm{SrF}_{2}: \mathrm{Yb} @ \mathrm{SrF}_{2}: \mathrm{Y}$ (12.2 nm) LNPs@Y (see Figure 1; Figure S3 in the Supporting Information) dispersed in water and therefore it has stable spectral properties. These LNPs and LNPs@Y remain dispersed for months with no sign of precipi-tation or agglomeration of the LNPs, which remain uniform and clear (see Figure 1). Figure 1i schematically illustrates the average dimensions of the core and the shell of the LNPs@Y. The size estimation by dynamic light scattering (DLS) and from transmission electron microscopy (TEM) images of the LNPs and the LNPs@Y can be found in Section S2 of the Supporting Information.

During this work, the presence of IPIE in the HLNFs is monitored through the energy transfer from $\mathrm{Nd}^{3+}$ to $\mathrm{Yb}^{3+}$ ions, as schematized in Figure 2a. The possibility of using the energy transfer as an indicator of IPIE is based on the fact that such energy transfer only occurs when donor $\left(\mathrm{Nd}^{3+}\right)$ and acceptor
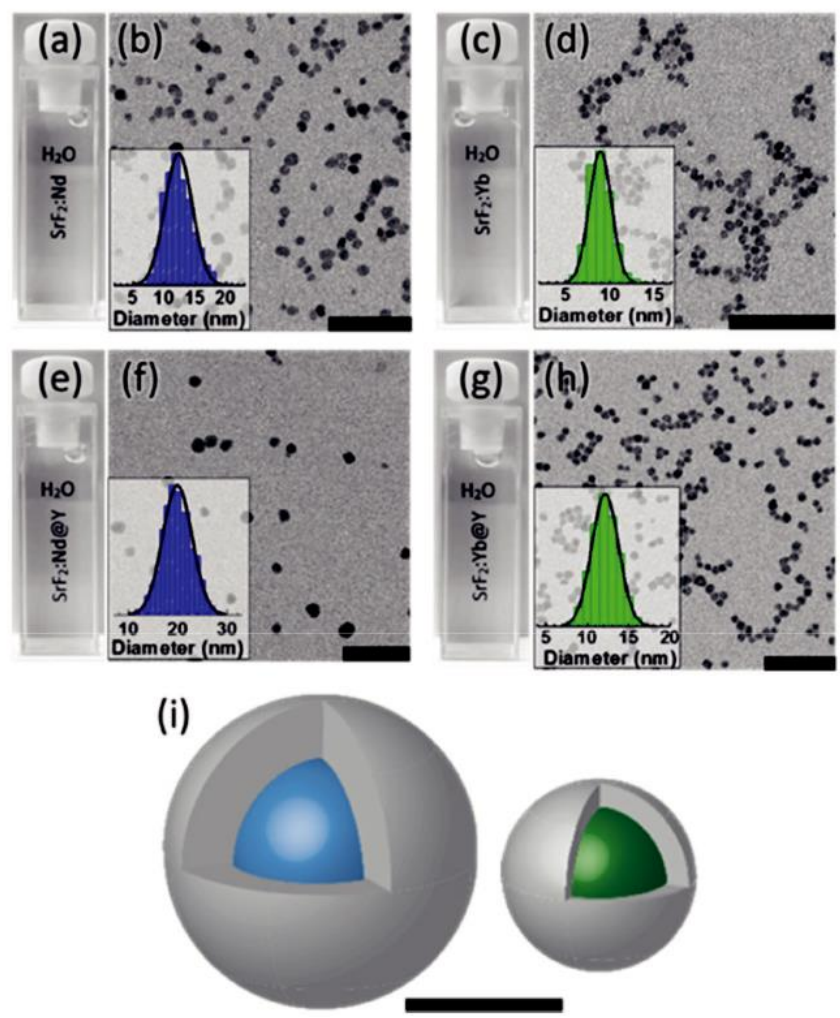

Figure 1. a), c), e), and g) Optical images of cuvettes containing the LNF:Nd, LNF:Yb, LNF:Nd@Y, and LNF:Yb@Y, respectively. b), d), f), and

h) TEM images and corresponding size distributions of $\mathrm{SrF}_{2}: \mathrm{Nd}, \mathrm{SrF}_{2}: \mathrm{Yb}$, $\mathrm{SrF}_{2}: \mathrm{Nd} @ \mathrm{SrF}_{2}: \mathrm{Y}$, and $\mathrm{SrF}_{2}: \mathrm{Yb} @ \mathrm{SrF}_{2}: \mathrm{Y}$ LNPs, respectively. TEM scale bars: $100 \mathrm{~nm}$. i) Schematic representation of the $\mathrm{SrF}_{2}: \mathrm{Nd@SrF}: Y$ (blue: Nd-doped core, grey: $\mathrm{SrF}_{2}: \mathrm{Y}$ shell) and $\mathrm{SrF}_{2}: \mathrm{Yb} @ \mathrm{SrF}_{2}: \mathrm{Y}$ (green: Yb-doped core, grey: $\mathrm{SrF}_{2}: Y$ shell) LNPs@Y.Scale bar: $10 \mathrm{~nm}$.

$\left(\mathrm{Yb}^{3+}\right)$ ions become close enough. In our case this means that when $\mathrm{Nd}^{3+}$ ions are optically excited the luminescence gener-ated by $\mathrm{Yb}^{3+}$ ions is only observed when both $\mathrm{Nd}^{3+}$ and $\mathrm{Yb}^{3+}$ ions are at the surface of the same LNP. This would only be possible if IPIE is taking place. Figure $2 b$ includes the emission of the original (homogeneous) luminescent nanofluids (LNFs) upon selective excitation of $\mathrm{Nd}^{3+}$ ions at $790 \mathrm{~nm}$. The emis-

sion of $\mathrm{Nd}^{3+}$ ions at around $1060 \mathrm{~nm}\left({ }^{4} \mathrm{~F}_{3} / 2 \rightarrow{ }^{4} \mathrm{I}_{11 / 2}\right)$ is observed from the LNF containing $\mathrm{SrF}_{2}: \mathrm{Nd}$ LNPs (LNF:Nd). As expected, excitation at $790 \mathrm{~nm}$ in a LNF containing only $\mathrm{SrF}_{2}$ :Yb LNPs (LNF:Yb) results in no emission $\left(\mathrm{Yb}^{3+}\right.$ ions show a negligible absorption cross section at this wavelength). Figure $2 \mathrm{~b}$ shows that when a HLNF is optically excited with a $790 \mathrm{~nm}$ beam the overall emission spectrum is constituted not only by the $\mathrm{Nd}^{3+}$ emission at $1060 \mathrm{~nm}$, but also by that of $\mathrm{Yb}^{3+}$ ions at $980 \mathrm{~nm}$

$\left({ }^{2} \mathrm{~F}_{5 / 2} \rightarrow{ }^{2} \mathrm{~F} 7 / 2\right)$. The presence of the emission generated by $\mathrm{Yb}^{3+}$ ions reveals the presence of $\mathrm{Nd}^{3+}$ to $\mathrm{Yb}^{3+}$ energy transfer and, therefore, constitutes an evidence of the existence of IPIE in the HLNF (see illustration in Figure 2c).

Just after the mixing of the LNFs to form the HLNF, the luminescence obtained from the acceptor ions $\left(\mathrm{Yb}^{3+}\right)$ by energy transfer is null, as shown in Figure 3a. Data included in Figure 3a show how the emission generated by acceptor ions increases with time until the saturation. This clearly indicates 
(a)

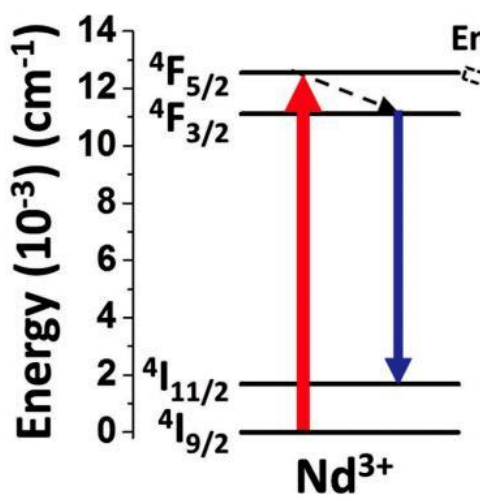

Energy transfer

(b)

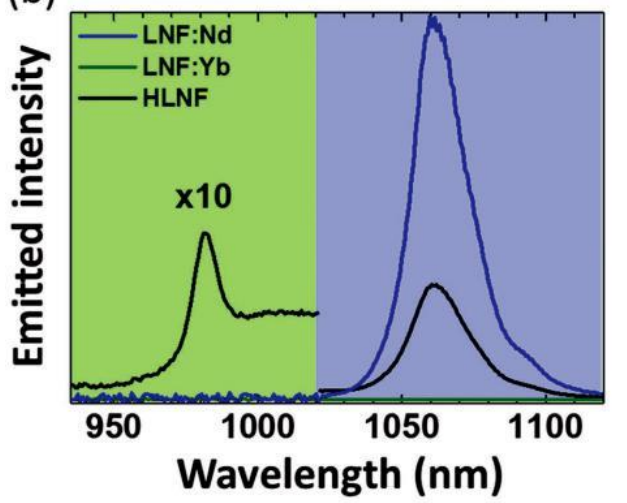

(d)
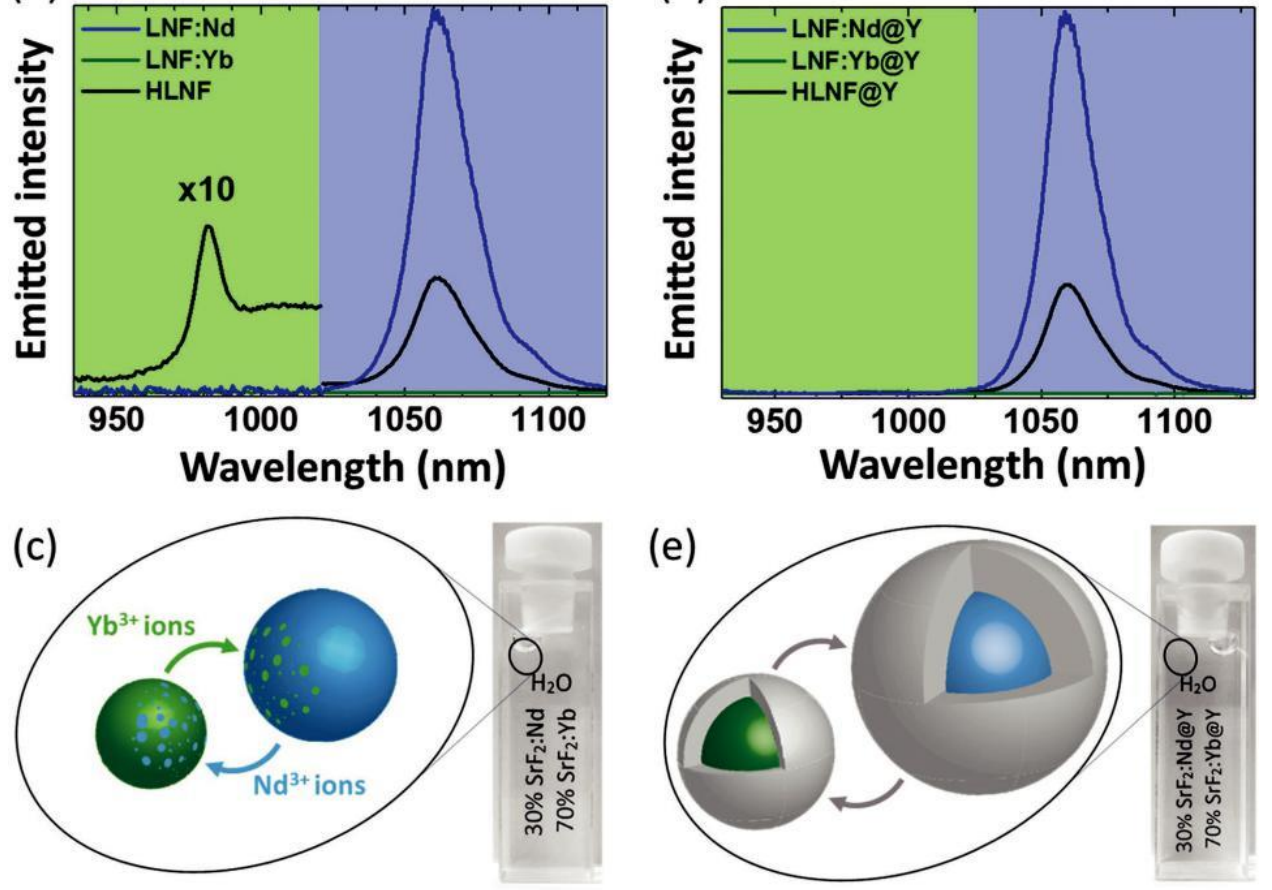

Figure 2. a) Energy levels diagrams of $\mathrm{Nd}^{3+}$ and $\mathrm{Yb}^{3+}$ ions pointing out the transitions involved in excitation (red arrow), energy transfer (dashed arrow), and emissions (blue arrow: $\mathrm{Nd}^{3+}$; green arrow: $\mathrm{Yb}^{3+}$ ). b) Emission spectra of LNF:Nd (blue), LNF:Yb (green), and HLNF after the saturation of IPIE due to long-term evolution at $50{ }^{\circ} \mathrm{C}$ (black) under selective excitation of $\mathrm{Nd}^{3+}$ ions at $790 \mathrm{~nm}$. Green and blue backgrounds delimit the emission band of $\mathrm{Yb}^{3+}$ and $\mathrm{Nd}^{3+}$ ions, respectively. c) Schematic illustration of IPIE process between LNPs and optical image of a cuvette containing the HLNF.

d) Emission spectra of LNF:Nd@Y (blue), LNF:Yb@Y (green), and HLNF@Y after the saturation of the IPIE due to long-term evolution at $50{ }^{\circ} \mathrm{C}$ (black) under selective excitation of $\mathrm{Nd}^{3+}$ ions at $790 \mathrm{~nm}$. e) Schematic illustration of a collision between LNPs@Y, where the SrF $2: Y$ shell avoids IPIE and optical image of a cuvette containing the HLNF@Y.

that IPIE is an accumulative process, and that after a cer-tain time (days, in our case) the IPIE between LNPs:Nd and LNPs:Yb is stopped as an equilibrium situation is achieved. The presence of IPIE in our HLNFs has been confirmed by energy-dispersive Xray spectroscopy (EDX) of single LNPs con-stituting the HLNF after the saturation of the IPIE. EDX meas-urements reveal the simultaneous presence of both $\mathrm{Yb}^{3+}$ and $\mathrm{Nd}^{3+}$ ions in a single and isolated LNP (see Figure S4b in the Supporting Information). Note that initially LNPs added to the fluid contained only either $\mathrm{Nd}^{3+}$ or $\mathrm{Yb}^{3+}$ and that the simulta-neous presence of both ions in an individual LNP is an une-quivocal indicator of the existence of IPIE.
Note that IPIE between the LNPS in the HLNFs has been produced without altering the original size of the LNPs, as proved by TEM and DLS (see Figure 3b,c; Figure S2 in the Supporting Information). As a matter of fact, the ratio between the number of LNPs:Nd and LNPs:Yb in the HLNF after saturation of the IPIE (obtained from the fit of the size distribution in Figure $3 \mathrm{c}$ ) corresponds to the proportion of LNPs estimated initially in the HLNF (there are approximately $6 \mathrm{SrF}_{2}: \mathrm{Yb}$ LNPs for every one $\mathrm{SrF}_{2}$ :Nd LNP in the HLNF). This fact also confirms the size stability of the LNPs used all along this work and reflects that all the spectroscopic changes observed can be ascribed to IPIE and not to morphological changes of the LNPs. 

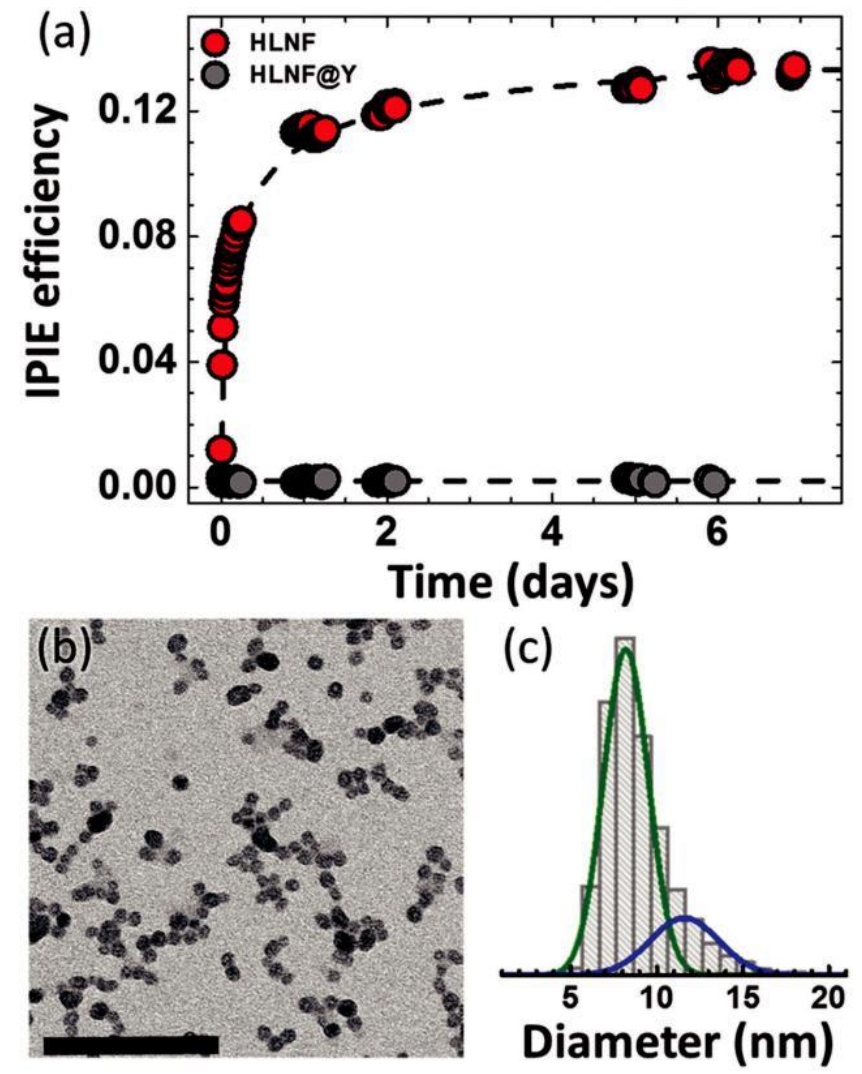

Figure 3. Long-term evolution of the IPIE efficiency of the HLNF (red dots) and of the HLNF@Y (grey dots) at $50{ }^{\circ} \mathrm{C}$. Black dashed lines are guides to the eye. b) TEM image and c) size distribution of the HLNF after long-term evolution at $50{ }^{\circ} \mathrm{C}$. Green and blue lines refer to the size distributions of $\mathrm{SrF}_{2}: \mathrm{Yb}$ and $\mathrm{SrF}_{2}: \mathrm{Nd}$ LNPs within the HLNF, respectively. Scale bar: $100 \mathrm{~nm}$.

The nature of the energy transfer process between the exchanged ions has been investigated by fluorescence lifetime measurements. Results are shown in Figure 4. It is found that

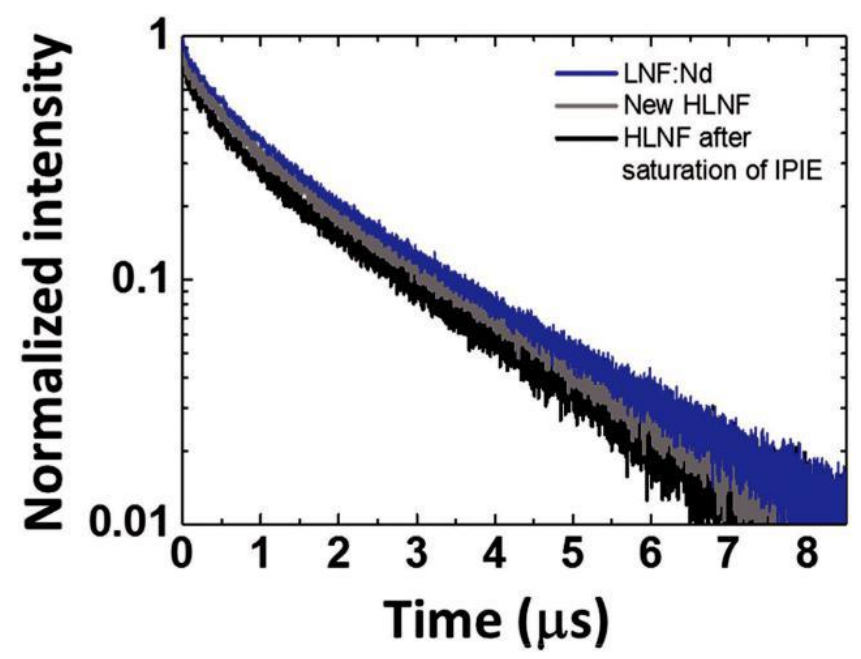

Figure 4. Intensity decay of the LNF:Nd (blue), the HLNF just after mixing (grey), and the HLNF after the saturation of the IPIE due to the long-term evolution at $50{ }^{\circ} \mathrm{C}$ (black) from ${ }^{4} \mathrm{~F}_{3 / 2}$ energy level (at $900 \mathrm{~nm}$ ) under excitation at $797 \mathrm{~nm}$. the decay time of the donor $\left(\mathrm{Nd}^{3+}\right)$ ions in the HLNF decreases with time as the IPIE takes place. Indeed, the fluorescence lifetime of neodymium ions in the HLNF decreases by almost $20 \%$ when saturation of the IPIE is reached (comparing with the lifetime obtained from LNF:Nd). Such reduction in the donor lifetime suggests that the energy transfer process is a nonradia-tive one. This was, indeed, expected as the nonradiative char-acter is characteristic of energy transfer processes between ions in close proximity, as those that are located in the surface of a same LNP after IPIE. Finally, it should be mentioned that the fluorescence lifetime of $\mathrm{Nd}^{3+}$ ions just after mixing the LNFs to form the HLNF is already reduced in comparison with the lifetime of LNF:Nd. This fact suggests that IPIE is taking place from the very first time that $\mathrm{SrF}_{2}: \mathrm{Nd}$ and $\mathrm{SrF}_{2}: \mathrm{Yb} \mathrm{LNPs}$ coexists in the same HLNFs, in accordance with the results included in Figure 3a. Note also that the ion exchange processes that lead to the loss of spectral stability are probably not only produced between $\mathrm{Nd}^{3+}$ and $\mathrm{Yb}^{3+}$ ions, but also between $\mathrm{Nd}^{3+} / \mathrm{Yb}^{3+}$ ions and $\mathrm{Sr}$ ions. This process has no effect on the luminescence of the LNPs, as in fact the luminescent ions are always replacing to $\mathrm{Sr}$ ions in the matrix. On the contrary, F ions are probably not taking part in this process, as the exchange between the luminescent ions and $\mathrm{F}$ ions should be evidenced by a change in the crystalline field felt by the luminescent ions and so the shape of the spectra of their emission should be altered.

Regarding the ability of the LNPs to interact between them, the role of the fluid surrounding the LNPs has to be consid-ered. In the particular case of water-based HLNFs, the tem-perature has a drastic effect on the interaction between water molecules and between water molecules and LNPs. Such inter-action decreases over certain crossover temperature due to the temperature induced change in the molecular state of water. This molecular crossover in the interaction between water mol-ecules is reflected in many temperature-dependent properties of water and is also related to the denaturation of proteins due to the increase of temperature. ${ }^{[13]}$ Below the crossover tempera-ture (about $45{ }^{\circ} \mathrm{C}$, see Figure 5), water molecules are capable of interacting between them forming sort of cages surrounding and isolating the LNPs. These cages of water molecules par-tially impede the IPIE and so the total saturation of this process is not achieved (see Section S3 in the Supporting Informa-tion). However, above the crossover temperature, the interac-tion strength between water molecules is strongly reduced and the formation of those "protective cages" is not possible. As a result, above the crossover temperature, water molecules cannot act as isolating agents of the LNPs, leading to a clear enhancement in the IPIE, as shown in Figure 5. In this figure, it is shown how for a nonsaturated HLNF the IPIE efficiency is almost temperature independent below the crossover tem-perature. However, above this temperature, the IPIE efficiency shows an astonishing increase with temperature. Once the IPIE takes place and modifies permanently the spectral properties of the HLNFs, they become less temperature dependent, as it is observed in the variation of IPIE efficiency during the cooling procedure. Thus, the IPIE is quickly nonreversibly saturated (see Figure 3a) for temperatures higher than that of the crossover. ${ }^{\text {[9] }}$ Experimental data included in Figure 5 reveal that, due to IPIE, the spectral stability of HLNFs is drastically affected by the molecular structure of water and, hence, by the 


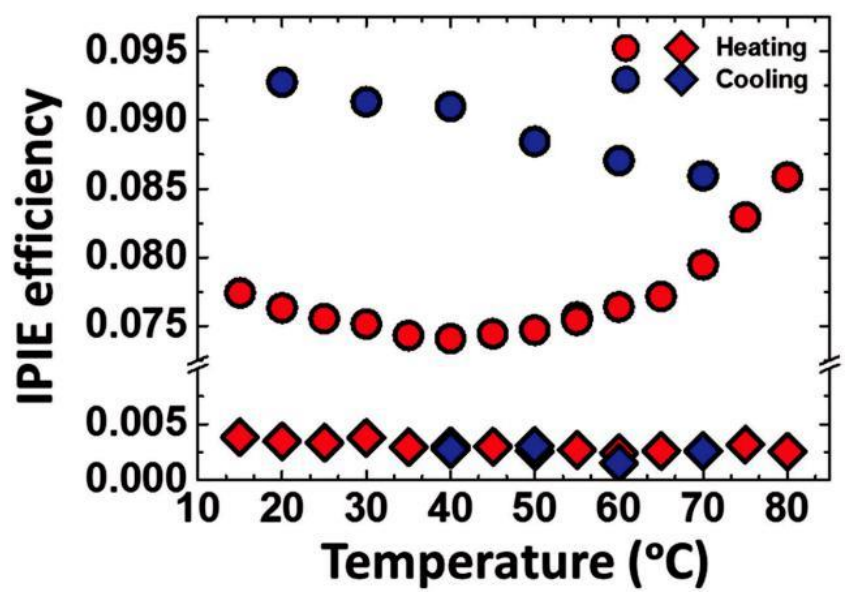

Figure 5. Temperature dependence of the IPIE efficiency of the HLNF (circles with black border) and HLNF@Y (circles with gray color) after long-term evolution at $20^{\circ} \mathrm{C}$ (red color for heating cycle, blue color for cooling).

medium temperature. Moreover, these nonreversible evolutions of the LNPs in the LNF (Figures 5 and 3a) evidence that we are not dealing with an interparticle energy transfer process (fluo-rescence resonant energy transfer), but indeed with permanent changes of the doping of the LNPs, which can be ascribed to the IPIE process.

The lack of both temporal and thermal spectral stability in HLNFs can be avoided by the addition of a nonlumines-cent shell to the LNPs surface, as schematically illustrated in Figure 2e. The spectrum of the HLNF@Y under selective excitation of $\mathrm{Nd}^{3+}$ ions is included in Figure 2d. No evidence of fluorescence from $\mathrm{Yb}^{3+}$ ions is found in these spectra. This indicates the absence of any $\mathrm{Nd}^{3+} \rightarrow \mathrm{Yb}^{3+}$ energy transfer, i.e., the lack of neodymium and ytterbium ions in close proximity. This fact unequivocally demonstrates how the presence of the nonluminescent shell in both $\mathrm{SrF}_{2}: \mathrm{Yb}$ and $\mathrm{SrF}_{2}: \mathrm{Nd}$ LNPs com-pletely avoids the IPIE of luminescent ions. $\mathrm{Sr}$ and $\mathrm{Y}$ ions in the nonluminescent isolating shell may experience ion exchange between shells of different LNPs@Y, but this process cannot be assessed by the luminescence of the LNPs@Y. The homogeni-zation in the HLNF is avoided by using this surface passiva-tion strategy even at longterm and at temperatures above the crossover of water molecules interaction, as shown in Figures $3 \mathrm{a}$ and 5.

Although the IPIE process is perfectly observed in water-based LNFs, the emission of the acceptor $\left(\mathrm{Yb}^{3+}\right)$ ions is quenched and absorbed by water (water shows an absorption band around the emission of $\left.\mathrm{Yb}^{3+}\right){ }^{[14]}$ For this reason, a more sensitive observation of the IPIE was expected from $\mathrm{D}_{2} \mathrm{O}$-based HLNF (DHLNF), as its absorption in that range is negli-gible. ${ }^{[14,15]}$ As shown in Figures S5b and S6 in the Supporting Information, the intensity emitted by the acceptor ions and the observed IPIE efficiency are approximately one order of mag-nitude higher than those of water-based HLNFs, although the time of saturation of the IPIE remains similar (compare those figures with Figures $2 b$ and $3 \mathrm{a}$, corresponding to water-based HLNFs). As expected, a crossover in the interaction between $\mathrm{D}_{2} \mathrm{O}$ molecules is also evidenced above about $50{ }^{\circ} \mathrm{C}$ (see
Figure S9a in the Supporting Information). As it was observed for the water-based HLNF, this D-HLNF undergoes no IPIE if a nonluminescent shell is grown over the LNPs to obtain a coreshell D-HLNF (D-HLNF@Y), as expounded in Section S3 in the Supporting Information. The energy transfer enabled by the IPIE is also nonradiative in the case of D-HLNF, as proved by the shortening of the intensity decays included in Figure S10 in the Supporting Information. In addition, the emission of the donor ion seems to be also less quenched by $\mathrm{D}_{2} \mathrm{O}$, as it shows a much larger lifetime in this case (see Table S2 in the Sup-porting Information). Thus, the energy transfer to the acceptor ions is increased as well, resulting in a larger decrease of the donor ion lifetime.

\section{Conclusion}

The loss of the spectral stability of the heterogeneous lumi-nescent nanofluid consisting of $\mathrm{SrF}_{2}: \mathrm{Nd}$ and $\mathrm{SrF}_{2}: \mathrm{Yb}$ nano-particles dispersed in water was studied in depth. It has been experimentally demonstrated how the luminescent properties of such heterogeneous nanofluids are strongly influenced by both time and temperature. Time and temperature induced instabilities are here unequivocally correlated to the presence of energy transfer processes that appear as a consequence of the exchange of surface ions between different nanoparticles. It is here demonstrated how in heterogeneous luminescent nanofluids both time and temperature induced spectral insta-bilities can be simultaneously avoided by adding a nonlumines-cent shell. This protective shell isolates the luminescent ions of the different nanoparticles and prevents their exchange. Core- shell engineering is here demonstrated as a unique tool for the development of stable heterogeneous nanofluids in which luminescent properties become independent on temperature, time, and molecular status of medium.

\section{Experimental Section}

Synthesis of the LNPs: The employed LNPs and LNPs@Y were synthesized by a hydrothermal method. ${ }^{[16]}$ A detailed description of the reagents and synthesis procedure can be found in Section S1 of the Supporting Information.

Spectroscopy Setup: (Emission) The emission spectra were obtained upon excitation at $790 \mathrm{~nm}$ by a single-mode fiber-coupled laser diode. The laser light was collimated into the home-made microscope by a fiber port. Then it was focused using a microscope objective lens (20x, NA = $0.4)$, which also collects the emission of the LNPs. The emission was selected by means of a wavelength selective mirror and a $830 \mathrm{~nm}$ longpass filter. Finally, the luminescence of the LNPs was analyzed by a spectrometer and recorded by an InGaAs detector. (Temperature) The temperature of the HLNFs was controlled by a heating plate $\left(0.1{ }^{\circ} \mathrm{C}\right.$ of accuracy). The temperature was modified at a rate of $2.5^{\circ} \mathrm{C} \mathrm{min}-1$. To ensure the homogeneous distribution of the heat in the cuvette containing the HLNF, the temperature was held and stabilized for $15 \mathrm{~min}$ before each measurement. The thermal gradient across the cuvette was under $1{ }^{\circ} \mathrm{C}$. (Lifetime) The intensity decay of the luminescence of the donor ions in the LNFs was obtained by exciting the ions at $797 \mathrm{~nm}$ by means of an optical parametric oscillator. The light emitted at around $900 \mathrm{~nm}\left({ }^{4} \mathrm{~F}_{3} / 2 \rightarrow{ }^{4} \mathrm{I} / 2\right)$ by the LNFs was selected by a spectrometer. Then this emission was detected and amplified by a photomultiplier tube (R636-10, Hamamatsu) and recorded by a digital oscilloscope. Note that 
the lifetime measured corresponded to the same energy level originating the $1060 \mathrm{~nm}$ emission band $\left({ }^{4} \mathrm{~F}_{3 / 2} \rightarrow{ }^{4} I_{11 / 2}\right)$.

Characterization Setup: The equipment and procedure employed to characterize the size, structure, and composition of the samples are described in detail in Section S1 of the Supporting Information.

\section{Acknowledgements}

This work was supported by the Spanish Ministerio de Educación y Ciencia (MAT2016-75362-C3-1-R) and by COST Action CM1403. L.L.$P$. thanks the Universidad Autónoma de Madrid for the "Formación de Personal Investigador (FPI-UAM)"program. P.H.-G. thanks the Spanish Ministerio de Economia y Competitividad for the Juan de la Cierva program (IJCl-2015-24551). M.P. and A.S. thank University of Verona (Italy) for financial support in the framework of the "Cooperint 2016" and "Ricerca di Base 2015" projects. The work of K.S. was supported by Latvian National Research Program IMIS2 (Grant No. 302/2012).

\section{Conflict of Interest}

The authors declare no conflict of interest.

\section{Keywords}

core-shell nanoparticles, lanthanide, nanofluids, water

[1] a) C. A. S. Batista, R. G. Larson, N. A. Kotov, Science 2015, 350, 1242477; b) W. Yu, H. Xie, J. Nanomater. 2012, 2012, 1.

[2] S. P. Jang, S. U. Choi, J. Heat Transfer 2007, 129, 617.

[3] B. del Rosal, D. H. Ortgies, N. Fernández, F. Sanz-Rodríguez, D. Jaque, E. M. Rodríguez, Adv. Mater. 2016, 28, 10188.

[4] C. D. Brites, X. Xie, M. L. Debasu, X. Qin, R. Chen, W. Huang, J.Rocha, X. Liu, L. D. Carlos, Nat. Nanotechnol. 2016, 11, 851.
[5] L. M. Maestro, P. Haro-González, M. C. Iglesias-De La Cruz, F. SanzRodríguez, Á. Juarranz, J. G. Solé, D. Jaque, Nanomedicine 2013, 8, 379.

[6] C. Yu, J. Irudayaraj, Anal. Chem. 2007, 79, 572.

[7] D. Geißler, L. J. Charbonnière, R. F. Ziessel, N. G. Butlin, H. G. Löhmannsröben, N. Hildebrandt, Angew. Chem., Int. Ed. 2010, 49, 1396.

[8] a) F. Wang, R. Deng, J. Wang, Q. Wang, Y. Han, H. Zhu, X. Chen, X. Liu, Nat. Mater. 2011, 10, 968; b) S. Sarkar, B. Meesaragandla, C. Hazra, V. Mahalingam, Adv. Mater. 2013, 25, 856.

[9] L. Labrador-Páez, D. J. Jovanovic', M. I. Marqués, K. Smits, S. D. Dolic', F. Jaque, H. E. Stanley, M. D. Dramic'anin, J. GarcíaSolé, P. Haro-González, D. Jaque, Small 2017, 13, 1700968.

[10] S. Han, X. Qin, Z. An, Y. Zhu, L. Liang, Y. Han, W. Huang, X. Liu, Nat. Commun. 2016, 7, 13059.

[11] J. Li, S. Schachermeyer, Y. Wang, Y. Yin, W. Zhong, Anal. Chem. 2009, 81, 9723.

[12] a) R. Ghosh Chaudhuri, S. Paria, Chem. Rev. 2011, 112, 2373; b) E. C. Ximendes, U. Rocha, C. Jacinto, K. U. Kumar, D. Bravo, F. J. López, E. M. Rodríguez, J. García-Solé, D. Jaque, Nanoscale 2016, 8, 3057; c) K. Prorok, M. Pawlyta, W. Strek,A. Bednarkiewicz, Chem. Mater. 2016, 28, 2295; d) J. Zuo, Q. Li, B. Xue, C. Li, Y. Chang, Y. Zhang, X. Liu, T. Langping, H. Zhang, X. Kong, Nanoscale 2017, 9, 7941.

[13] a) J. G. Davis, K. P. Gierszal, P. Wang, D. Ben-Amotz, Nature 2012, 491, 582; b) L. Maestro, M. Marqués, E. Camarillo, D. Jaque, J. G. Solé, J. Gonzalo, F. Jaque, J. C. D. Valle, F. Mallamace, H. Stanley, Int. J. Nanotechnol. 2016, 13, 667; c) F. Mallamace, C. Corsaro, H. E. Stanley, Sci. Rep. 2012, 2, 993; d) J. C. del Valle, E. Camarillo, L. Martinez Maestro, J. A. Gonzalo, C. Aragó, M. Marqués, D. Jaque, G. Lifante, J. G. Solé, K. Santacruz-Gómez, Philos. Mag. 2015, 95, 683; e) P. Gallo, K. Amann-Winkel, C. A. Angell, M. A. Anisimov, F. d. r. Caupin, C. Chakravarty, E. Lascaris, T. Loerting, A. Z. Panagiotopoulos, J. Russo, Chem. Rev. 2016, 116, 7463.

[14] M. Quintanilla, I. Cantarelli, M. Pedroni, A. Speghini, F. Vetrone, J. Mater. Chem. C 2015, 3, 3108.

[15] R. Arppe, I. Hyppänen, N. Perälä, R. Peltomaa, M. Kaiser, C. Würth, S. Christ, U. Resch-Genger, M. Schäferling, T. Soukka, Nanoscale 2015, 7, 11746.

[16] a) M. Pedroni, F. Piccinelli, T. Passuello, S. Polizzi, J. Ueda, P. Haro-González, L. Martinez Maestro, D. Jaque, J. García-Solé, M. Bettinelli, Cryst. Growth Des. 2013, 13, 4906; b) I. Villa, A. Vedda, I. X. Cantarelli, M. Pedroni, F. Piccinelli, M. Bettinelli, A. Speghini, M. Quintanilla, F. Vetrone, U. Rocha, Nano Res. 2015, 8, 649; c) S. Zanzoni, M. Pedroni, M. D'Onofrio, A. Speghini, M. Assfalg, J.Am. Chem. Soc. 2015, 138, 72. 